\title{
A New Rotator Interval Approach for Antegrade Humeral Nailing: A Technical Note
}

\author{
Naxin Liu1, Toshikazu Mohri², Junzou Hayashi², Ryo Orito², Tsuyoshi Nakai², \\ Yasushi Yoneda ${ }^{1}$ \\ ${ }^{1}$ Department of Orthopaedic Surgery, Takasago Seibu Hospital, Hyogo, Japan \\ ${ }^{2}$ Department of Orthopaedic Surgery, Itami City Hospital, Hyogo, Japan \\ Email: naxin2jp@yahoo.co.jp
}

Received 14 September 2014; revised 31 October 2014; accepted 4 November 2014

Copyright (C) 2014 by authors and Scientific Research Publishing Inc.

This work is licensed under the Creative Commons Attribution International License (CC BY).

http://creativecommons.org/licenses/by/4.0/

c) (7)

\section{Abstract}

The lateral and anterior lateral approaches are the most commonly used for antegrade nailing of humerus fractures. However, the surgical exposure is restricted by the bony acromion. The iatrogenic injury to the rotator cuff can also cause post-operative pain and compromise shoulder function. This article describes a new rotator interval approach that we used for central entry point nailing. In this approach, the skin incision starts from the midpoint between the acromion and coracoid process. A trans-rotator interval split in front of the anterior border of the supraspinatus (SSP) tendon is then made to open the glenohumeral joint. With the SSP retracted laterally and the long head of the biceps (LHB) retracted medially, the humeral head is directly visualized. The entry point can thus be determined and confirmed by intra-operative fluoroscopy in both axial and AP planes. We recommend this rotator interval approach as an alternative nailing technique for 2-part humeral neck fractures and humeral shaft fractures.

\section{Keywords}

\section{Antegrade Humeral Nailing, Approach, Rotator Interval}

\section{Introduction}

Antegrade intramedullary nailing has been proven to be an effective surgical method in treating humeral neck and humeral shaft fractures. Comparing with dynamic compression plating, it involves less invasive incision, preservation of the periosteum around the fracture and lower rate of infection [1]. Good or excellent results have been reported with the use of the recent angular and sliding stable nails. Their high three-dimensional locking properties can provide reliable fixation in elderly osteoporotic patients [2] [3]. 
The standard approaches for antegrade nailing are the lateral and anterior lateral acromion approach [4] [5]. However, in both cases, surgeons struggle to operate in a restricted narrow working space under the bony acromion. In addition, they have to incise the hypovascular tendon part of the rotator cuff, which may lead to postoperative shoulder pain and functional impairment [6] [7]. Parks described a rotator interval approach to resolve the iatrogenic injury to the rotator cuff, but by using an antero-superior approach, long incision and wide soft tissue dissection were made to identify the rotator interval and entry point of the nail [8].

We managed to reach the humeral head through the rotator interval, by an original incision from the midpoint between the acromion process and the coracoid process. During operation, the inserting point can be kept under direct observation. Usually, a $3-4 \mathrm{~cm}$ skin incision is sufficient for the nail insertion.

\section{Surgical Technique}

The operation is performed under general anesthesia. The patient is placed in the beach-chair position with the operated shoulder protruded over the edge of the table. A pillow under the chest of the operated side is used to elevate the shoulder. The arm is reclined at the tableside, with the hand on an arm rest board. The shoulder joint is extended at approximately 40 degrees to make the incision site align with the humeral axis. A C-arm fluoroscopy machine is set above the head side of the operation table.

\subsection{Step 1: Skin Incision}

The midpoint between the anterior acromion corner and the coracoid process is marked. A 3 to $6 \mathrm{~cm}$ longitudinal incision is made from the marked point to the anterior part of the great tuberosity (Figure 1 and Figure 2).

\subsection{Step 2: Rotator Interval Confirmation}

After a blunt splitting of the deltoid muscle (Figure 3a), the subacromial bursa is incised. The anterior border of the supraspinatus tendon (SSP) is identified. Palpation of the great tuberosity and the bicipital groove can provide additional landmarks to identify this soft junction (Figure 2).

\subsection{Step 3: Joint Incision}

The shoulder joint is opened along the anterior border of the SSP. Long head of the biceps (LHB) can be seen directly under this incision (Figure $3 b$ ).

By retracting the LHB medially and the SSP laterally, the cartilage humeral head is exposed (Figure 3c).

\subsection{Step 4: Determine the Inserting Point}

The guide pin is inserted (Figure 3d). We use both axial and AP views of intra-operative fluoroscopy to confirm the position and direction of the guide pin (Figure 4).

\subsection{Step 5: Insert the Nail and Locking Screws}

(Figure 3e).

\subsection{Step 6: Rinse and Closure}

An end cap is inserted at last (Figure 3f). The humeral joint and the operation field are carefully rinsed to eliminate hematoma and bone debris residuals within the joint. The rotator interal and joint capsule is repaired with Vicryl 3.0 suture.

\subsection{Step 7: Intra-Operative X-Ray Photographs}

2 direction X-ray photographs are taken after wound closure (Figure 5).

\section{Postoperative Rehabilitation Schedule}

Codman pendulum exercise and painless passive ROM training are started from the next day after operation. The sling is kept for 4 weeks. Resistance exercise training begins after the bony healing is confirmed. 


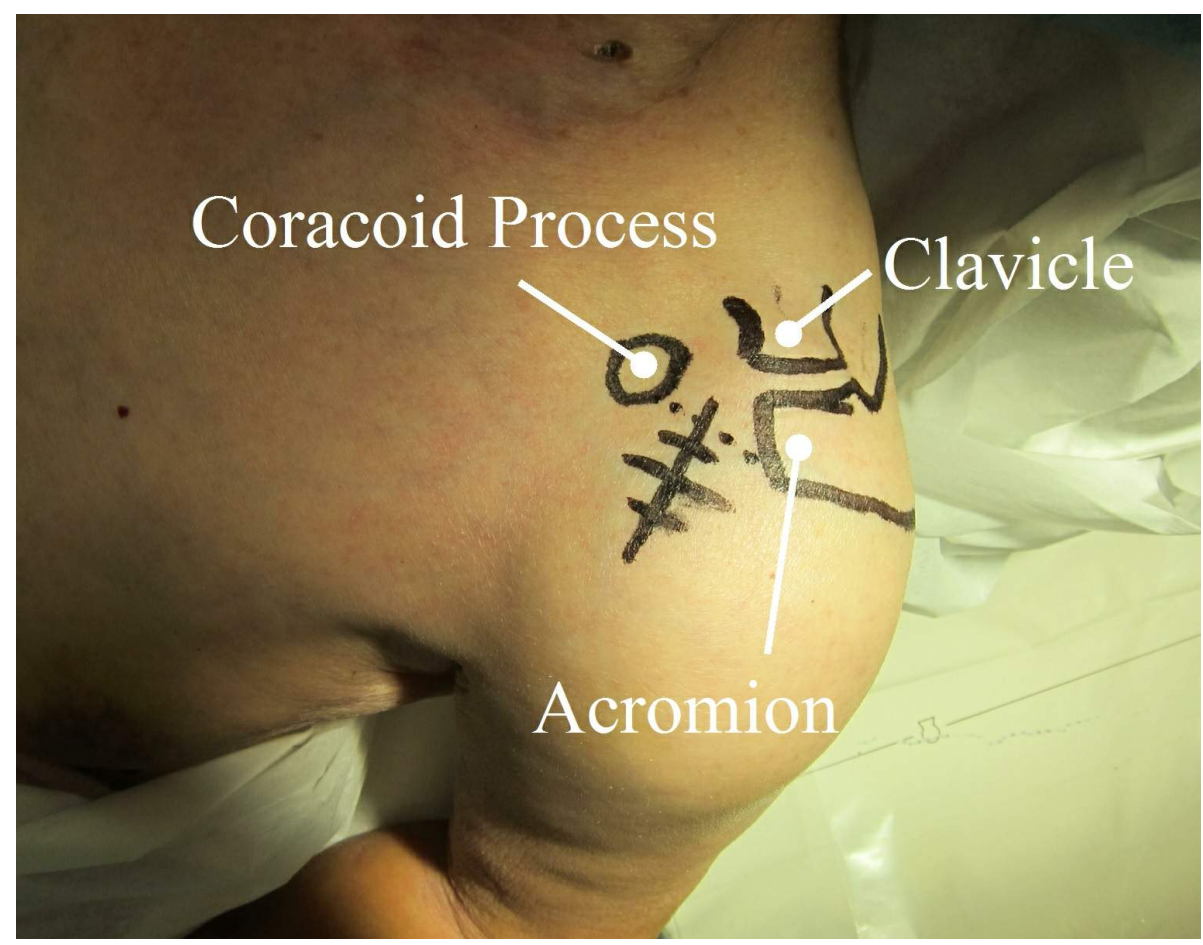

Figure 1. Patient position and skin marking (see Step 1).

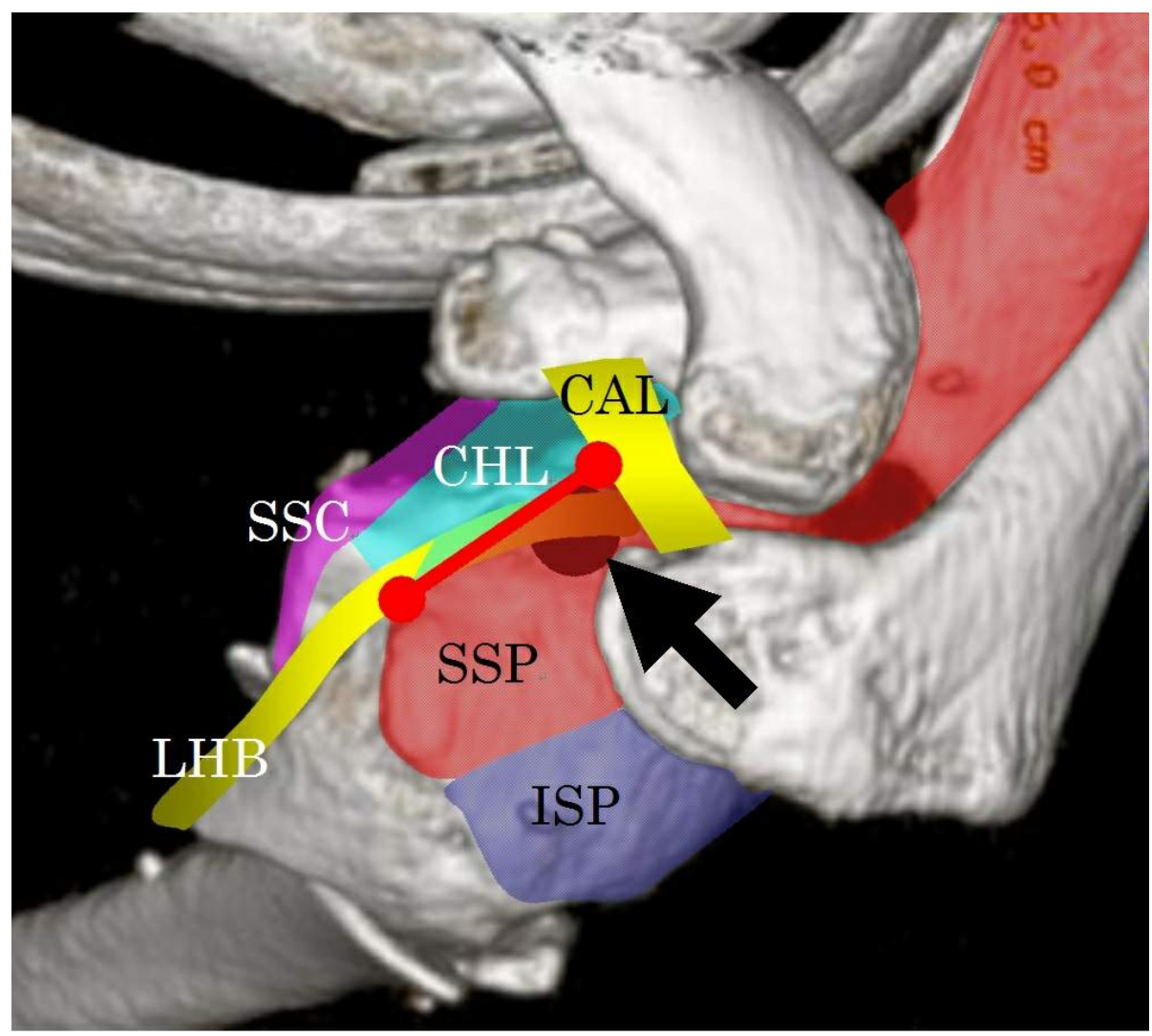

Figure 2. Rotator interval and schematic landmarks illustrated on the patient's 3D-CT. Red line: skin incision; Black arrow: nail entry hole; SSC: subscapularis; CHL: coracohumeral ligament; ISP: infraspinatus muscle; CAL: coraco acromial ligament. 


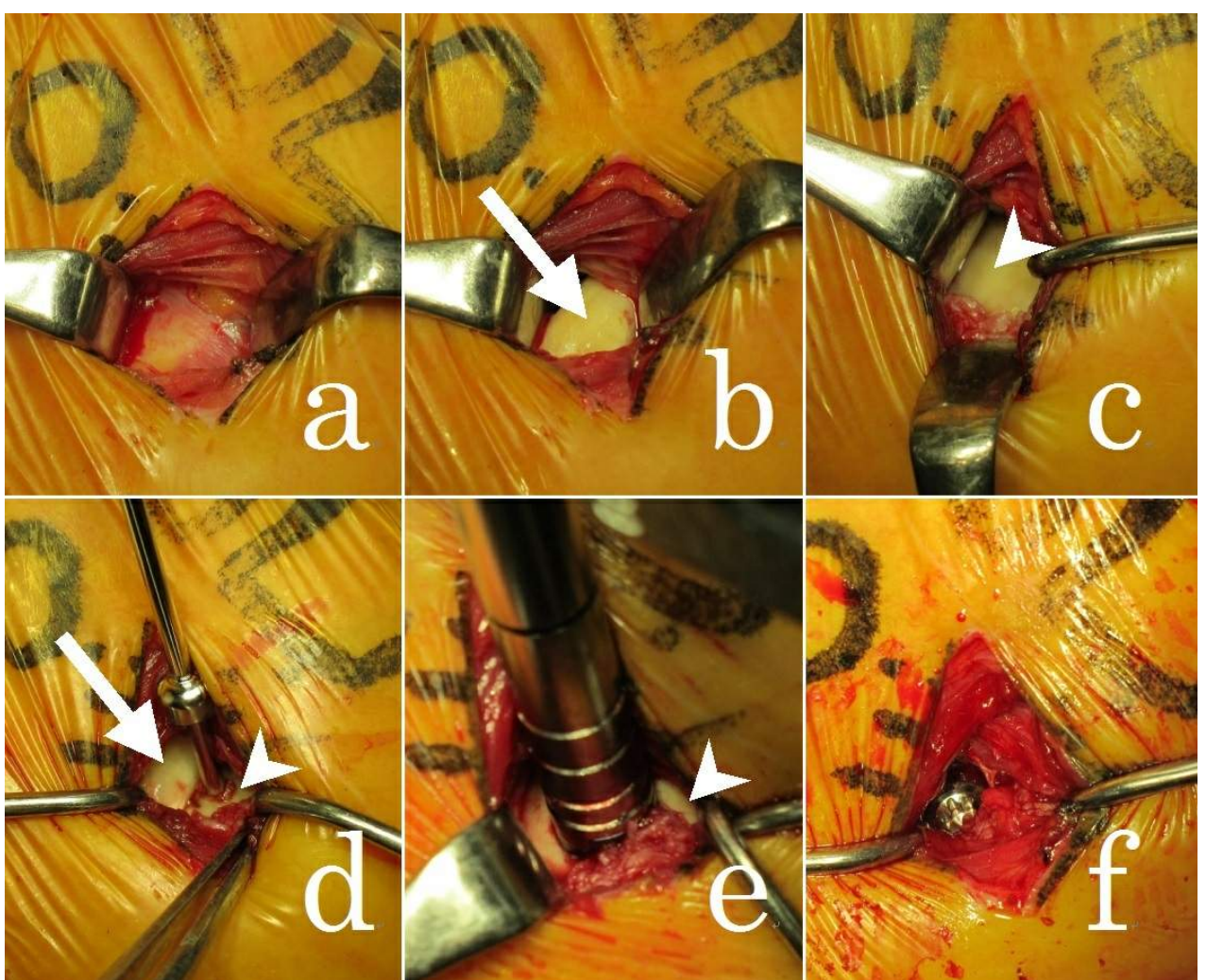

Figure 3. Surgical procedures in each step: a: skin incision and muscle splitting (see Step 2); b and c: LHB (arrow) and humeral head (arrowhead) under LHB (see Step 3); d: guide pin inserting point, and its relationship with LHB (arrow) and humeral head (arrowhead) (see Step 4); e: nail inserting into the humeral head (see Step 5); f: end cap finished (see Step 6).

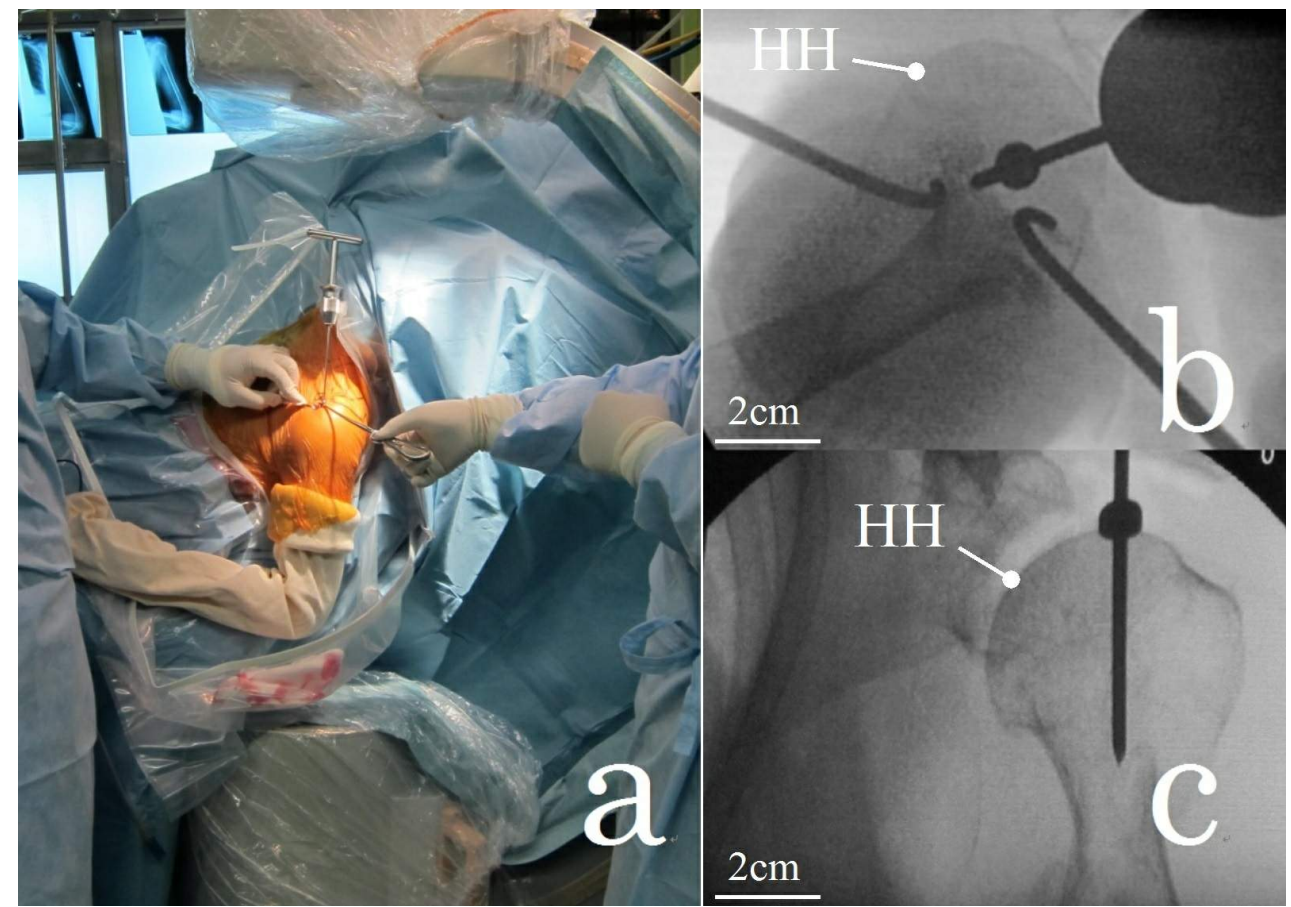

Figure 4. Intra-operative fluoroscopy confirm (see Step 7): a: C-arm position for axial view; b: axial view image; c: AP view image; $\mathrm{HH}$ : humeral head. 


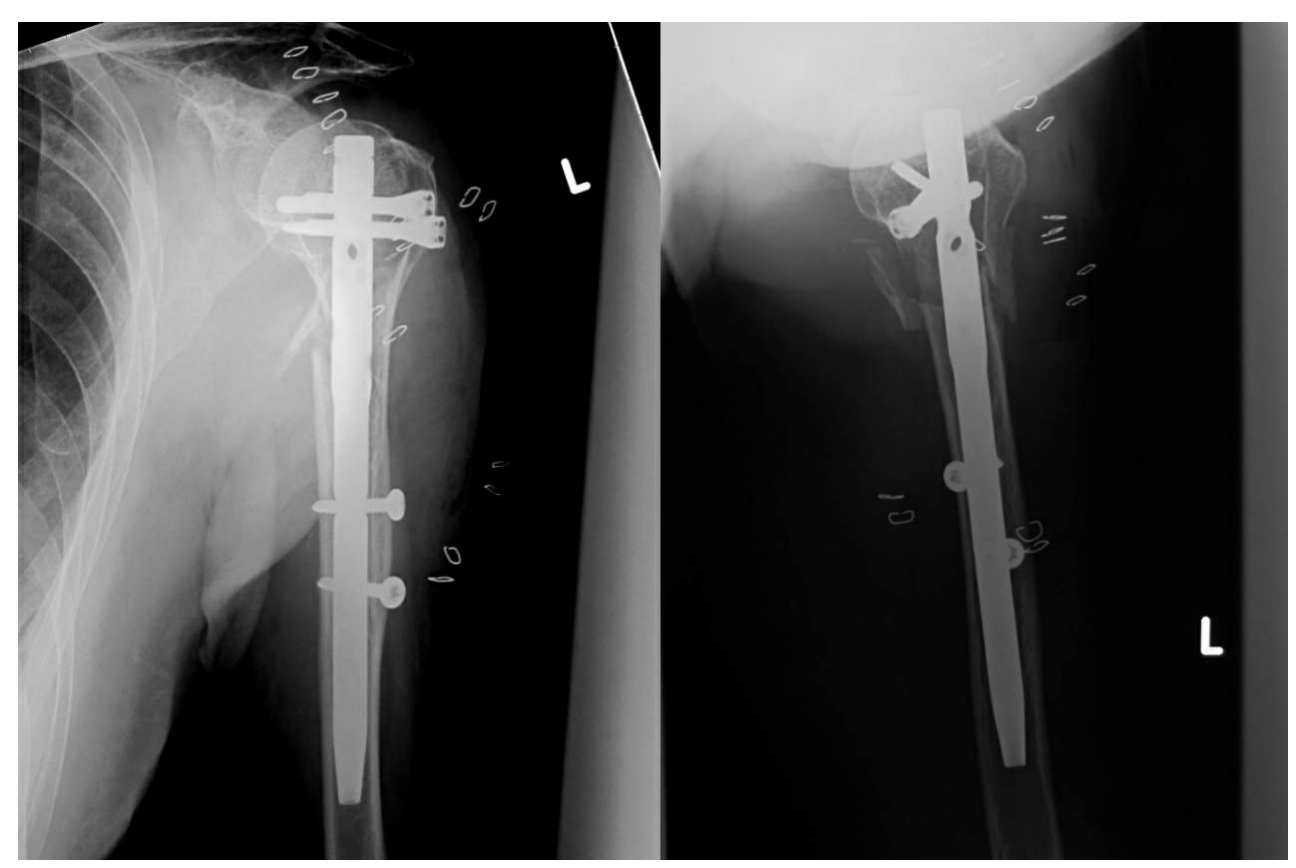

Figure 5. Intra-operative X-ray photography.

\section{Discussion}

The rotator interval is at the anterosuperior aspect of the glenohumeral joint. It is bordered superiorly by the supraspinatus, inferiorly by the subscapularis, and medially by the base of the coracoid process. The interval portion of the shoulder joint capsule is reinforced externally by the coracohumeral ligament (CHL), internally by the superior glenohumeral ligament (SGHL) and traversed by the intraarticular biceps tendon. Theoretically, because the trans-rotator interval approach represents a surgical split in the musculotendinous junction, it should not adversely affect cuff function. Park [8] reported for the first time a rotator cuff interval technique for the antegrade humeral nailing. He used the anterosuperior approach to the shoulder to reach the rotator interval. After dissection of the coracoacromal ligament, an incision between the posterior border of the long head of biceps and the anterior border of supraspinatus was made to reach the humeral head. Shiota [9] reported antegrade nailing after intra-operation arthroscope identification of the rotator interval. A $4 \mathrm{~cm}$ transverse incision between the anterior acromion and the coraccoal process was reported. The rotator interval incision was the same with Park. Excellent postoperative ROM and VAS score were reported in all his cases.

The skin incision of our approach is medial to the conventional lateral, anterolateral and Riemer's anterior approach [7]. Unlike the transverse incision described by Shiota, we made a longitudinal incision. It is parallel to the muscle fibers of deltoid and aligned with our rotator interval incision on the anterior border of supraspinatus. Positioning is the most important factor to get the acromion and the CAL out of the incision area, and to gain direct lineal access through the rotator interval to the humeral head. In all of our cases, the CAL was not transected.

In big and muscular patients, SSP can be stiff and difficult to move laterally. External rotation of the humeral head is then required to bring the entry point into the incision area. Proximal extension of the skin incision with resection of the CAL may also be applicable in difficult cases.

\section{Conclusion}

We find this rotator interval approach safe and effective to cope with 2-part humeral neck and humeral shaft fractures.

\section{Conflict of Interest Statement}

The authors declare that they have no conflict of interest related to the publication of this manuscript. 


\section{References}

[1] Lin, J. and Hou, S.M. (1999) Antegrade Locked Nailing for Humeral Shaft Fractures. Clinical Orthopaedics and Related Research, 365, 201-210. http://dx.doi.org/10.1097/00003086-199908000-00025

[2] Mittlmeier, T.W., Stedtfeld, H.W., Ewert, A., et al. (2003) Stabilization of Proximal Humeral Fractures with an Angular and Sliding Stable Antegrade Locking Nail. Journal of Bone and Joint Surgery, 85, 136-146.

[3] Gradl, G., Dietze, A., Arndt, D., Beck, M., Gierer, P., Börsch, T. and Mittlmeier, T. (2007) Angular and Sliding Stable Antegrade Nailing (Targon PH) for the Treatment of Proximal Humeral Fractures. Archives of Orthopaedic and Trauma Surgery, 127, 937-944. http://dx.doi.org/10.1007/s00402-007-0425-5

[4] Hoppenfeld, S., deBoer, P. and Buckley, R. (2009) Surgical Exposures in Orthopaedics: The Anatomic Approach. 4th Edition, Lippincott Williams and Wilkins, Philadelphia, 30-35.

[5] Canale, S.T. and Beaty, J.H. (2013) Campbell's Operative Orthopaedics. 12th Edition, Elsevier Mosby, Philadelphia, 2846-2848.

[6] Lin, J., Shen, P.W. and Hou, S.M. (2003) Complications of Locked Nailing in Humeral Shaft Fractures. The Journal of trauma, 54, 943-949. http://dx.doi.org/10.1097/01.TA.0000032252.57947.47

[7] Riemer, B.L., D’Ambrosia, R., Kellam, J.F., Butterfield, S.L, and Burke 3rd, C.J. (1993) The Anterior Acromial Approach for Antegrade Intramedullary Nailing of the Humeral Diaphysis. Orthopedics, 16, 1219-1223.

[8] Park, J.Y., Pandher, D.S., Chun, J.Y. and Md, S.T. (2008) Antegrade Humeral Nailing through the Rotator Cuff Interval: A New Entry Portal. Journal of Orthopaedic Trauma, 22, 419-425. http://dx.doi.org/10.1097/BOT.0b013e318173f751

[9] Shiota, Y., Mogami, A. and Kirimura, K. (2012) Antegrade Humeral Nailing through the Rotator Interval. Katakansetsu, 36, 477-480. (In Japanese) 
Scientific Research Publishing (SCIRP) is one of the largest Open Access journal publishers. It is currently publishing more than 200 open access, online, peer-reviewed journals covering a wide range of academic disciplines. SCIRP serves the worldwide academic communities and contributes to the progress and application of science with its publication.

Other selected journals from SCIRP are listed as below. Submit your manuscript to us via either submit@scirp.org or Online Submission Portal.
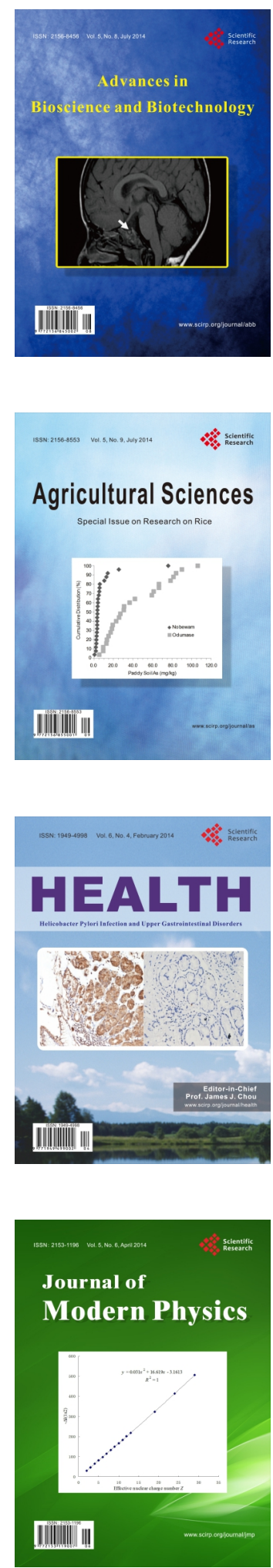
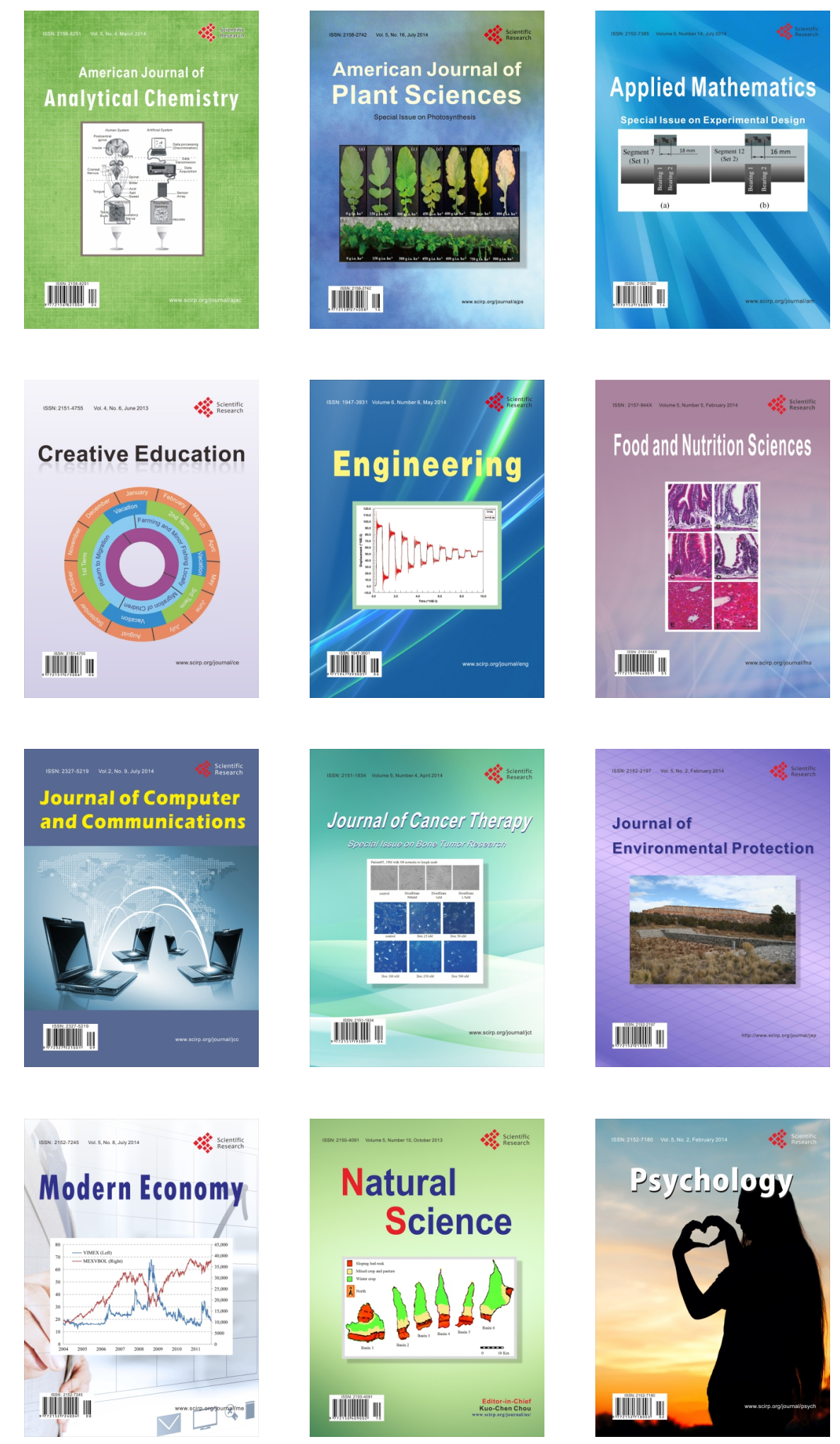